\title{
Research on the Leisure Cross-Country Orienteering of the Park in the Fitness of the Elderly
}

\author{
Linbao Zhang \\ P.E Department, Shangrao Normal University, Shangrao 334000, China. \\ 3235777167@qq.com
}

Keywords: leisure cross-Country orienteering of the park, elderly, fitness.

\begin{abstract}
This paper uses the literature material law, field observation, the research methods, such as visiting survey method, in shangrao young to the old city as the research object, by the means of combining theory and practice research on the elderly leisure park on foot orientation for study. The results show that there are realistic conditions for the leisure orientation of the elderly park. Park leisure orientation is necessary in elderly people; The recreational orientation of the park can promote the health of the elderly in many ways. Park leisure orientation in the elderly requires a certain strategy. Through this study aims to: based on the background of an aging society, explore the park leisure walking orientation in the elderly fitness timing, necessity, etc., to carry out diversified development to provide reference for the elderly fitness.
\end{abstract}

\section{Research object definition}

The WHO defines the age of the elderly as: 60-74 years old for young people, 75-89 years old for the aged, and over 90 years old for the elderly. Object of this research mainly is young to the old, the age is in the transition from the old adaptation period, physiological and psychological comes at a sensitive stage, is very important to whether life happiness for the rest of the elderly. Therefore, it is worthwhile to explore scientific and reasonable sports intervention to promote healthy and happy old people. This study screened young people in shangrao city, jiangxi province, 50 males and 50 females. Method of selection: use the early publicity of the week to carry out voluntary registration.

\section{Park leisure hiking orientation definition.}

Directional movement according to the different classification standard has many forms, this research defines: leisure park on foot orientation is directional (a type of directional cross-country) on foot, is with the aid of ones and map, in the park to complete the intended target activities by way of aerobic exercise. The leisure foot orientation movement can give full play to the brain dominance function, the coordination of the limbs and reaction to the brain, promoting the harmonious and healthy development of the organism.

\section{Management analysis of physical exercise for the aged.}

Researchers are used to the concept of "sportsman" because it is a good way to explain the important role of sports in changing people. Through the interview survey found that $95 \%$ of elderly people think, the association of sports organization and management is not perfect, the innovation of physical exercise methods, lack of enthusiasm and inputs such as project, which directly or indirectly affect the effect of physical exercise in the elderly. The survey also found that there are still a lot of elderly people who are "physically active", lack of scientific training methods and effective exercise programs. Effective organization and scientific management will play an important role in promoting the physical exercise effect of the elderly and ensuring the health of the elderly. 


\section{Analysis of the conditions for the orientation of leisure walking in the elderly park.}

\subsection{Environmental improvement}

In recent years, governments at all levels have started to improve the urban environment, especially the construction and improvement of a series of venues suitable for urban residents' leisure activities. City presents a lot of artificial and natural fusion modern characteristic leisure places, such as give yiyang city forest park, park, entrepreneurial culture park, public parks, leisure park on the sanjiang park, etc. The existence of these parks provides the natural conditions for the elderly people to walk in the park.

\subsection{Policy and regulatory support.}

With the coming of the age of aging in China, the government has introduced a series of policies and measures to care for the elderly, which has laid a humane condition for the sports activities of the elderly. The relevant regulations of our country have explicit provisions on the sports fitness rights and interests of the elderly. Articles 16 and 46 of the sports law provide relevant provisions to ensure the development of sports activities for the elderly. "The protection of old people's rights stipulated in article 32:" the state and society shall adopt measures to carry out the mass cultural, sports and recreational activities suitable for the elderly, the elderly spiritual and cultural life of the rich." Article 8 of the national fitness regulations: the development of a national fitness program and a national fitness program should give full consideration to the special needs of students, the elderly, the disabled and rural residents. Promulgated by the state general administration of sports in 2000 elderly sports development plan "pointed out: to adapt to an ageing society demand for sports work, meet the old people's growing sports demand, follow from place to place, because, when they intend and scientific principle of civilization, and play sports in the elderly life rich and promote social stability and civilization, efforts for the elderly to create a scientific, civilized, and convenient to the nearest fitness environment.

\section{Park leisure foot orientation is necessary in the fitness of the elderly.}

\subsection{The diversified development needs of elderly fitness programs.}

With the need for physical fitness among the elderly, the selection of diversified fitness programs has become an important part of the fitness movement of the elderly. Continuous research and development of new and practical physical fitness programs for the elderly has become a necessity.

\subsection{The arrival of China's aging society and the existence of empty nesters.}

The arrival of an aging society means a large increase in the proportion of elderly people in China and an increase in the number of empty nesters. How to protect the physical and mental health of the elderly and promote the development of a harmonious society is an important task before us. Therefore, it is the best choice to continuously excavate the fitness content and form of old people and improve the quality of life of the elderly.

\section{Park leisure foot orientation for the health promotion of the elderly.}

\subsection{It is helpful to prevent senile dementia.}

Studies have shown that the causes of alzheimer's disease are complex, and lack of physical and mental exercise is one of the causes. Recreational hiking orientation is the brain, and body together to participate in activities of a body of intellectual sport, its body get event at the same time, let the elderly can also be fully active in the elderly brain, to maintain good brain function, must have effect on alzheimer's disease prevention and rehabilitation.

\subsection{Increase the sense of achievement for the elderly.}

There are many checkpoints for recreational hiking, and the elderly get a sense of success when 
they arrive at each checkpoint. A sense of accomplishment that translates into "old and useful" is helpful for the mental health of the elderly.

\subsection{Reducing the loneliness of the elderly and enhancing the happiness of the elderly.}

Recreational hiking directional collective project, can promote to participate in the communication between team members, is advantageous to the old people belonging to meet, this can greatly reduce the elderly loneliness, improve the old happiness, improve the quality of life.

\subsection{Reduce the incidence of cerebrovascular disease in the elderly.}

The study suggests that cardiovascular disease is mainly caused by overabundance of lipids, alcohols and other substances in the diet, and the lack of reasonable exercise promotes the metabolism of these substances. Park leisure orientation on foot is a very good aerobic exercise, be interesting, stimulating, can arouse the enthusiasm of the elderly activities, for the elderly, leisure walking directional moderate intensity, set up suitable for the elderly population of the collective project, scientific and reasonable, can guarantee the rationality of exercise in the elderly, which can reduce the incidence of cerebrovascular disease in the elderly.

\section{Park leisure foot orientation in the elderly population fitness strategies.}

\subsection{The dissemination of the basic knowledge and practical skills of casual hiking.}

In the park, we can set the basic common sense, basic technology, operation skill and other publicity column of the leisure hiking, and expand the awareness of the orientation of leisure hiking. We will give special lectures on leisure orientation and raise the awareness of leisure orientation.

7.2 Adopt the transplantation method to integrate other projects with leisure orientation, and develop a park orientation program suitable for the physical condition of the elderly.

In the leisure orientation movement of the elderly, it is possible to combine the sports activities of the elderly, such as walking and cross-country walking, with the orientation movement. On the basis of promoting the fun of directional movement, the "life vitality" of directional movement is broadened.

\subsection{The leisure hiking orientation of the elderly park is carried out in the form of team cooperation.}

In the old age group, the cooperative form of leisure orientation is adopted, the first can achieve the mutual help and protection of the team members, and ensure the safety; The second can promote mutual trust and emotional communication, and satisfy the happiness of the elderly, especially the empty nesters.

\subsection{Establishment of the local elderly park leisure foot orientation service station dominated} by the government.

The orientation service station can organize the various activities of leisure walking orientation; It can protect the lawful rights and interests of the elderly.

\subsection{Park leisure foot orientation project selection and venue setting should be in line with the actual situation of the elderly.}

Comprehensive consideration of the elderly fitness and psychological needs, leisure walking directional choose route and checkpoint more project design is relatively simple, such as "star" (the starting point and end point in the same place, similar to the geometric center of the field, the radiated around checkpoints around this point. In the course of the game, the player returns to the center every time he arrives at a checkpoint, and so on, and so on. ; Along the routes; The route is designed to avoid the mountain and the hilly, which are too steep and large, so as to avoid endangering the health of the elderly. The number of checkpoints is not easy, and it is recommended to be around 10-15. 


\subsection{Park leisure orientation should be carried out in the elderly for the purpose of "leisure and fitness".}

Leisure fitness is different from competitive sports, the sports load is small, to control within the scope of the bull 's-eye rate, movement time limit can be approximately 40 minutes is given priority to, so that we can very good to improve the index of the elderly health fitness.

\subsection{Carry out regional cooperation model for leisure orientation activities and exchanges in the elderly park.}

The regional cooperation model can draw lessons from the good operation mode of the leisure orientation of the old people's park. It can promote the exchange of old people in different regions and improve the value space of the elderly. Regional cooperation can also expand the thinking space and innovate new forms of leisure orientation.

\subsection{To give full play to the role of local colleges and universities targeted students, to provide services for the elderly orientation movement.}

As the curriculum reform unceasingly thorough, the local colleges and universities sports professional according to the requirements of the diversified personnel training, curriculum development, directional movement has become many current college sports professional courses, and many colleges and universities have established the directional sports teams. This can give play to the cooperation between institutions and local communities, so that students majoring in orienteering can provide guidance to the elderly orientation movement regularly, so as to facilitate the scientific development of orienteering in the elderly.

\section{Summary}

The aging society, empty nesters and other human social phenomena urgently need to pay attention to the elderly group, among which the research of health intervention means is indispensable. The recreation of the park is oriented to the practical needs, providing a diversified choice for the physical exercise of the aged and providing the conditions for the healthy body and mind. It is a good choice for the health intervention of the elderly, which is characterized by excellent sports environment, controllable exercise intensity and body intelligence. Therefore, it is a good thing for the health and sports service of the elderly to develop the best mode of the park leisure walking.

\section{References}

[1] Linbao Zhang. Sports entertainment [M]. Beijing: China books press, 2016.

[2] Xiaowei Zhang. Orienteering [M]. Beijing: star map publishing house, 2013.

[3] Yuanli Zhu,Bo Gou. Guidance of national fitness activities [M]. Xi 'an: Shaanxi science and technology press, 2011.

[4] Zhongren Hou. Analysis of directional movement culture [J]. Journal of sports culture, 2015, (12): 58-61. 